\title{
Rapid and Efficient Synthesis of Silver Nanofluid Using Electrical Discharge Machining
}

\author{
Kuo-Hsiung Tseng, ${ }^{1}$ Heng-Lin Lee, ${ }^{1}$ Chih-Yu Liao, ${ }^{1,2}$ \\ Kuan-Chih Chen, ${ }^{1}$ and Hong-Shiou Lin ${ }^{1}$ \\ ${ }^{1}$ Department of Electrical Engineering, National Taipei University of Technology, Taipei 10608, Taiwan \\ ${ }^{2}$ Light Source Division, National Synchrotron Radiation Research Center, Hsinchu 30076, Taiwan
}

Correspondence should be addressed to Kuo-Hsiung Tseng; khtseng@ee.ntut.edu.tw

Received 5 January 2013; Accepted 31 March 2013

Academic Editor: Jian Wei

Copyright (C) 2013 Kuo-Hsiung Tseng et al. This is an open access article distributed under the Creative Commons Attribution License, which permits unrestricted use, distribution, and reproduction in any medium, provided the original work is properly cited.

\begin{abstract}
The electrical discharge machining (EDM) system has been proven feasible as a rapid and efficient method for silver nanofluid preparation. This study prepared the silver nano-fluid via EDM and investigated the relationship between its process parameters and product characteristics. The prior study had found that the silver nano-fluid prepared by EDM contained both silver nanoparticles and silver ions. Silver ions had revealed the cause of the high suspension of the silver nanoparticles. To examine the relationship between the stability of silver nanofluid and the process parameters, this study quantified the relationship of process parameters to the material removal rate (MRR) of silver electrode and silver ion output rate (IOR) in the fluid, in order to achieve the most effective process parameter condition. Furthermore, the stability of silver nano-fluid was analyzed by various devices, including UV-Vis spectroscopy, size-distribution, and Zeta-potential analyzer. The effects of MRR, IOR, particle size, Zeta-potential, and optical properties of silver nanofluid under different process parameters are also discussed.
\end{abstract}

\section{Introduction}

Silver nanofluid has the potential for broad applications. The most typical application of nanosilver is its antibacterial effect, which has been proved to be able to inhibit various kinds of bacteria. Its commercial products can be widely seen on the market. Among the various methods to prepare silver nanofluid, this study employed the electrical discharge machining (EDM) system. The EDM system uses concentrated energy to sputter the electrode surface in dielectric fluid, and this method has been widely applied in nanofabrication technology, for example, $\mathrm{Ni}-\mathrm{C}$ powders in pure ethanol [1], silver nanofluid in ethylene glycol [2], gold nanoparticles in water or ethanol [3], and carbon nanotubes [4]. The EDM method has already been discussed by Ichinose et al. [5], and furthermore, Tien et al. [6] discovered that the resultant silver nanofluid fabricated by EDM method not only contains silver nanoparticles but also ionic silver [7], both can apply in the sterilization, for example, Staphylococcus aureus, and already tested by Tien et al. [8]. In addition, for commonly nanofluid applications, the thermal conductive material, like diamond nanofluid [9], $\mathrm{Al}_{2} \mathrm{O}_{3}$ [10], carbon nanotubes [11], $\mathrm{TiO}_{2}$ [12], $\mathrm{Cu}$ [13], $\mathrm{Ag}$ [14], and so forth nanofluids, the thermal conductive properties had discussed, since the particle concentration is high and size in particle is low which results in good quality of thermal conductivity behavior [15]. For nanofluid application issue, nanofluid must be strongly stable which does not sediment before application or analysis, but many factors may affect the stability of suspension, such as concentration of particles, particle size in diameter, solution properties, electric conductivity, and $\mathrm{pH}$ [16]. These factors enhance complex interactions to the nanofluid, for example, Van Der Waals combination force, electrostatic Coulomb repulsion force both were discussed in colloidal science DLVO (Derjaguin, Landau, Verwey, and Overbeek) theorem [17-19].

Literatures provide many methods in analyzing the stability of nanofluid. This study utilized the intensity of absorption 
TABLE 1: The key parameters of electrical discharge machining (EDM) for silver nanofluid production.

\begin{tabular}{lc}
\hline Parameters & Values \\
\hline Dielectric fluid volume & $200 \mathrm{~mL}(18.2 \mathrm{M} \Omega \cdot \mathrm{cm})$ \\
Anode diameter & $1 \mathrm{~mm}$ \\
Cathode diameter & $2 \mathrm{~mm}$ \\
Electrodes: metallic silver rod & $99.9 \%$ \\
Open circuit voltage & $140 \mathrm{~V}$ \\
Arcing current & $11.5 \pm 0.5 \mathrm{~A}$ \\
On-off duration & Variable: $5-5$ to $1000-1000 \mu \mathrm{s}$ \\
Temperature & Room temperature $\left(25^{\circ} \mathrm{C}\right)$ \\
Fabrication process time & $1,2,3 \mathrm{~min}$ \\
Pressure & $1 \mathrm{~atm}$ \\
\hline
\end{tabular}

spectrum to determine the stability of nanoparticle suspension in the bottle and further analyzed whether the electric potential at the surface of nanoparticle reached the stable level by Zeta-potential analyzer. Moreover, the size-distribution meter was employed to determine whether the samples in the bottle would be concentrated and augmented with the time variation. In the EDM process, the concentration of particles and ions would rise as the time increased, and the relationship affecting the stability was the key point for this study. The primary factor of well-suspension nanofluid is the maintenance of Zeta-potential [20-22]. The Zeta-potential represents the Coulomb repulsion force which prevents the particles from aggregating or precipitating out of solution. However, many factors like the particle size, particle/ion concentration, and $\mathrm{pH}$ could affect Zeta-potential magnitude or maintenance $[22,23]$. In this research, the EDM system was equipped with bulk silver rod (99.9\%), after a minute process in water; the resultant silver nanofluid contains both silver nanoparticles and ionic silver. In this case of analysis, both concentrations of the silver nanoparticles concentration and ionic silver concentration raised will enhance aggregating phenomenon, which defines as unstable suspension nanofluid [24-26]. Therefore, to evaluate nanofluid stability fabricated by EDM, the ideal way is to show the decay slope of UV-absorbance intensity of every sample and compare the Zeta-potential and average size of nanofluid [27, 28].

\section{Materials and Methods}

2.1. Preparation of Silver Nanofluid. In this study, the silver nanofluid was fabricated by EDM system, as shown in Figure 1. The EDM system contains an anode and cathode, which are both $99.9 \%$ silver rod, submerged in DI water $(200 \mathrm{~mL})$. The anode and cathode are chosen specifically in different diameter, because the anode removal rate is greater than cathode around 50 times [29], and the smaller anode aligns the plasma column which maintains on the electrodes surface plate not on the edge. The power supply supplies 140 Volts pulse DC on electrodes; the average arcing distance between electrodes is around $30 \mu \mathrm{m}$. The servo control system drives upper electrode (anode) to move down, that enables the electrons emitted from cathode to strike on anode surface

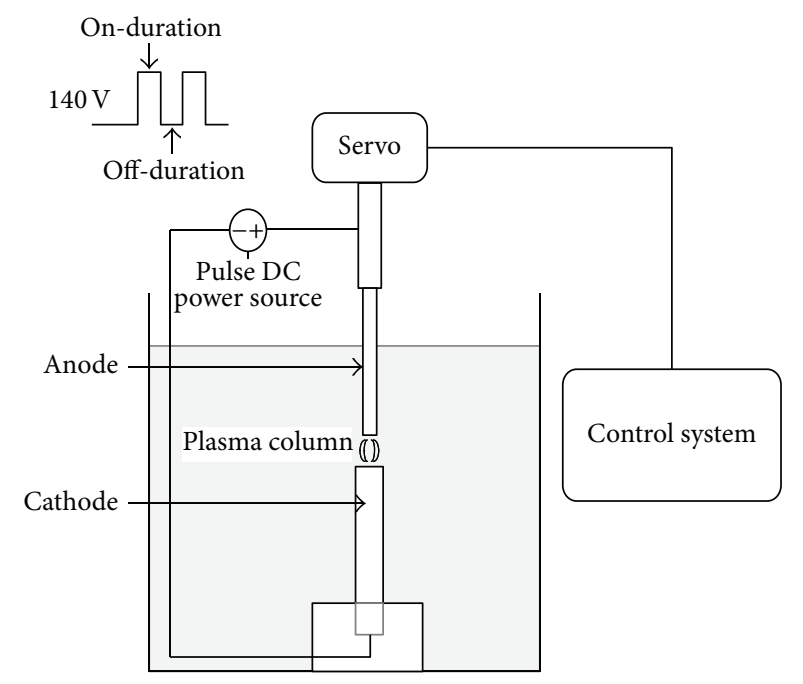

FIGURE 1: The scheme of electrical discharge machining (EDM) for silver nanofluid production.

continuously. Examination of the relationship between the characteristics of product and the process parameter (onoff duration) is the main purpose in this investigation. The detailed conditions are shown in Table 1.

2.2. Material Removal Rate (MRR) and Ion Output Rate (IOR). In electrical discharge machining processing, the arcing energy creates material removal rate (MRR, $\mathrm{mg} / \mathrm{sec}$ ) and ion output rate (IOR, $\mathrm{ppm} / \mathrm{sec}$ ), which are measured in different on-off time duration by electrode loss and titration (METTLER TOLEDO, DL50), respectively. MRR indicates measured anode loss after a significant process time (1 minute), because the main weight loss occurred on anode, and that the anode to cathode ratio is about 98:2 [29]; for this reason, we ignored the cathode loss while quantifying MRR. The relations between MRR and on-off time duration are nonlinear, which has been determined by [30, 31]. MRR and IOR are time dependent functions of EDM processing time.

2.3. Suspension Stability Test. After electrical discharge machining processing, the resultant silver nanofluid was collected, which contains sprayed silver nanoparticles and ionic silver, since the silver nanofluid fabricated by EDM was forming as a complex suspension fluid. There have been two main effects that will affect suspension stability, including the steric space and charged particle effects. Steric effect means higher concentration of nanofluid which will enhance particle collision and combination effect. Therefore, the crowded and negative charged silver nanoparticles will attract the positive charged ionic silver, and this makes the interaction in the resultant nanofluid becomes more complex. Like the charged particle-ion combination reaction and ions reduction reaction, then the suspension quality of nanofluid has been influenced. The factor explained by GouyChapman theory that the ionic silver concentration will arise while EDM process time increased [18]. The increasing of 


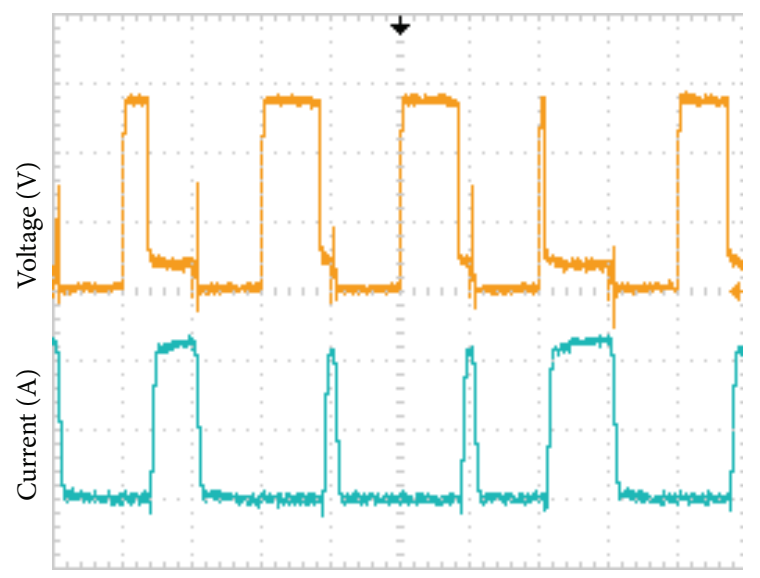

FIGURE 2: Voltage and current waveform during the EDM process.

ion concentration enables the compressing of double-layer Debye length; then the Zeta-potential magnitude gradually decayed. Finally, the suspension quality has been influenced. In addition, the concentration of suspension particle does affect Van Der Waals combination force. Thus the ionic silver concentration and the charged particle both can affect the suspension stability. In this study, the parameters of on-off duration were changed in order to investigate their influence on the process. The surface potential, size-distribution, and characteristic absorption peak value were analyzed by Zetapotential, size-distribution, and UV-Vis spectroscopy, and the stability of fluid samples was tested through long-time observation. For the process parameters of the samples, the on-off duration of IOR and MMR maximum value was selected as the experimental objects, and the saturated and unsaturated samples obtained by the total processing time of 1 and $3 \mathrm{~min}$, respectively, were analyzed.

\section{Results and Discussion}

3.1. Quantify the Discharge Energy. When the voltage applies to the electrode by a fixed on-off duration, not all cycles can discharge successfully. The measured electrical discharge is shown in Figure 2 (on-off duration, 30-30 $\mu \mathrm{s}$ ), where the voltage pulse generated 41 pulses in this period, but only 23 current pulses were found (i.e., successful electrical discharges), so the arcing rate was around 55\%. However, it is complicated to accurately quantify the discharge energy, with consideration of both the arcing rate (\%) and the arcing duration $\left(T_{\text {arc }}\right)$. Under the consideration of only the arcing rate, the oscilloscope was utilized to obtain the relative times of current signals produced by the electrical discharge machining, and the influence of different on-off durations to the arcing rate was compared. As shown in Figure 3, when the duration increased, the arcing times per second would be in proportion to the duration, theoretically; however, the experiment indicated that this slope was larger than the theoretical value. Thus, as presented in Figure 4, the arcing rate reached the maximum value at 10-10, 20-20, and 30$30 \mu \mathrm{s}$. That is to say, more arcs, more melted silver, could be produced at the settings of 10-10, 20-20, and 30-30 $\mu$ s.

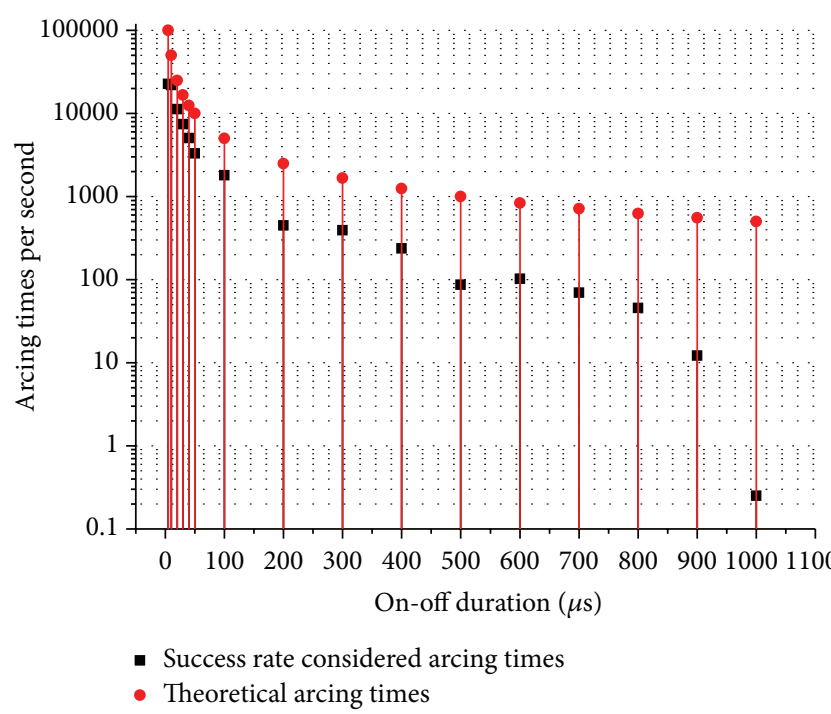

FIGURE 3: Comparison of theoretical and practical arcing times at different on-off duration.

3.2. Quantification of MRR and IOR. The concentration of particles and ions increased gradually along with time. Thus, the nanofluid contains more and more matters, results the change of steric space, ionic strength and $\mathrm{pH}$ value. The quantification result of MRR and IOR at different on-off duration (from 5 to 100) setting is shown in Figure 5. It shows that MRR and IOR were at the minimum when the on-off duration was set at 5-5 and 100-100 $\mu$ s, but they reached the maximum values when the on-off duration setting was at $30-30 \mu \mathrm{s}$. This is probably because that the best arcing rate could be obtained at 30-30 $\mu$ s, so MRR and IOR could reach their maximum values. High arcing rate represented that the energy accumulation and release have achieved the best fitting and were influenced by the electrode geometry, types of dielectric fluid, and magnitude of power source.

3.3. UV-Vis Analysis Results. For EDM process parameters, the on-off duration was set as $30-30 \mu$ s and the processing times as 1 and $3 \mathrm{~min}$; the two samples for UV-Vis spectrum were analyzed at $400 \mathrm{~nm}$. The analysis results are shown in Figure 6. The analysis method was time-based, where measurements were taken at the time $0 \mathrm{~h}, 12 \mathrm{~h}$, and $24 \mathrm{~h}$. The experiment revealed that the slope of sample was relatively low at $1 \mathrm{~min}$; the concentrations of samples of silver nanoparticle were still larger than $2 \mathrm{~min}$ and $3 \mathrm{~min}$ at $12 \mathrm{~h}$, and the concentrations of three samples were difficult to be measured at $24 \mathrm{~h}$, denoting that all silver nanoparticles were deposited; so in the samples of $2 \mathrm{~min}$ and $3 \mathrm{~min}$, the nanoparticle showed the trend of saturation.

3.4. Size-Distribution and Zeta-Potential. As shown in Figure 7, the samples of $1 \mathrm{~min}$ and $3 \mathrm{~min}$ were set at the same time points (settling for $10 \mathrm{~min}$ after the electrical discharge) for measurement. After settling for $10 \mathrm{~min}$, the particle size of 3 min sample $(\sim 1200 \mathrm{~nm})$ was larger than that of $1 \mathrm{~min}$ sample $(\sim 600 \mathrm{~nm})$; this is because $3 \mathrm{~min}$ sample 


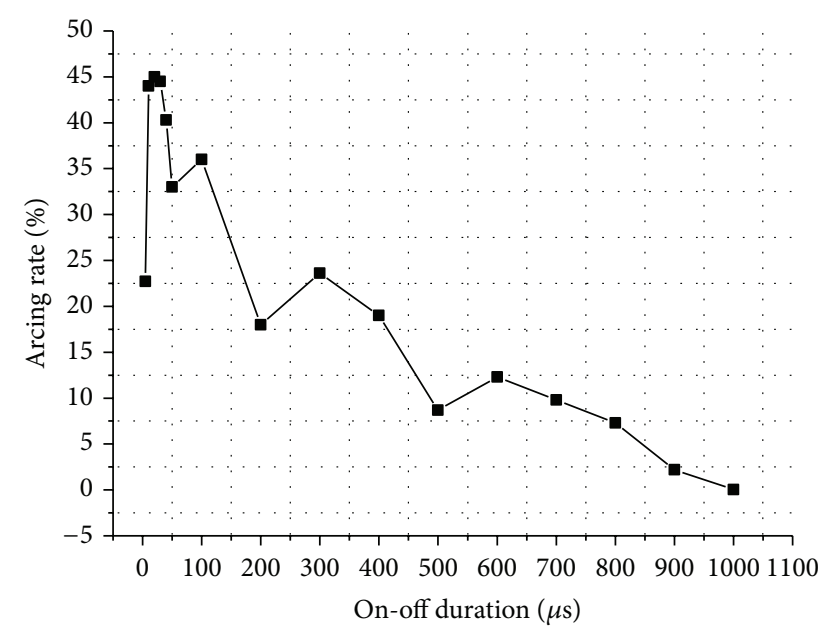

FIGURE 4: Arcing rate chart at different on-off duration.

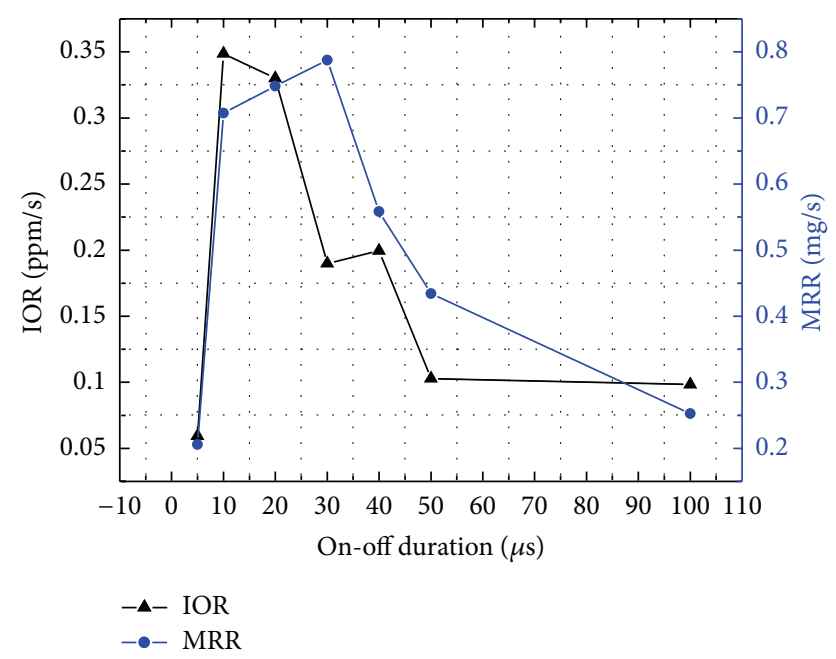

FIGURE 5: Material removal rate (MRR) and ion output rate (IOR) versus on-off duration setting.

was already saturated and aggregated during the electrical discharge. When taking the measurement after settling for $10 \mathrm{~min}$, the particle would aggregate for a period of time. After the continuous measurement for $50 \mathrm{~min}$, the particle sizes of $1 \mathrm{~min}$ and $3 \mathrm{~min}$ samples showed the trend of aggregation and becoming larger. The slope value of particle concentration of $3 \mathrm{~min}$ sample was slightly bigger than that of $1 \mathrm{~min}$ sample; hence, the $3 \mathrm{~min}$ sample particle would produce a large amount of aggregation and deposits during the electrical discharge. Since the particle concentration of 3 min sample while settling was greater than that of $1 \mathrm{~min}$ sample, the influence of concentration on the aggregation reaction could be known. After the EDM process, there were $\mathrm{Ag}$ ions and Ag particles in the dielectric fluid. The surface of Ag particles is charged (called Zeta-potential), whose magnitude dominated the suspension stability of particles. The continuous measurement of samples after settling for 15 55 min is illustrated in Figure 8. In the 3 min sample, due to greater concentration, the suspension space of charged

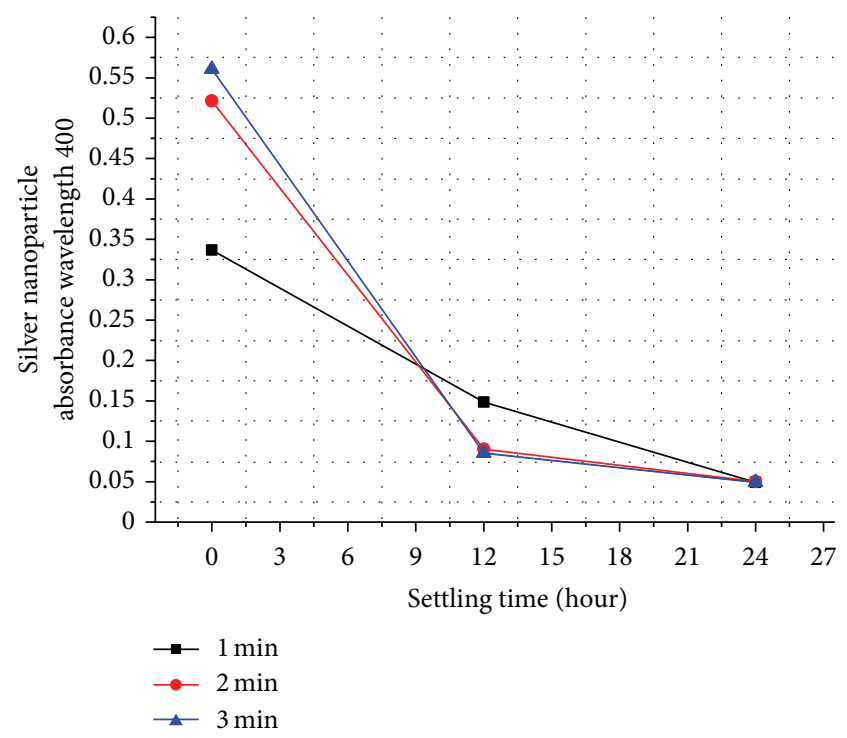

FIgURE 6: The UV-Vis absorbance of silver nanofluid at $400 \mathrm{~nm}$ versus settling time.

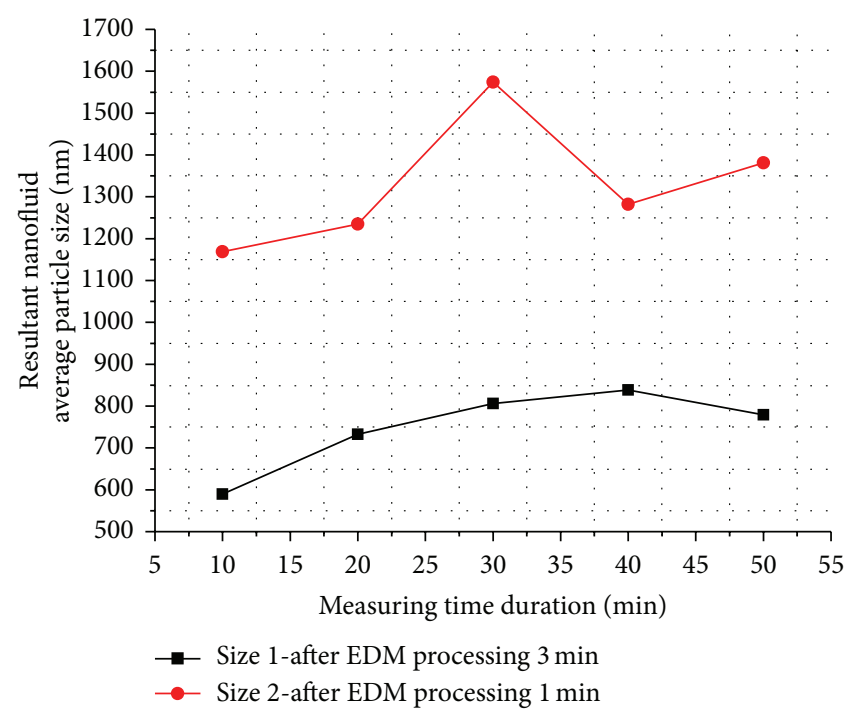

FIGURE 7: Particle size analysis of silver nanofluid.

particle was compressed; thus, the electrical double layer was compressed so that the Zeta-potential magnitude was lower than that of 1 min sample. However, through the observation of the slope of these two samples, it was found that Zetapotential magnitude increased with time, probably because the small particles with relatively high potential became prominent after big particles aggregated and deposited.

3.5. The influence of Different On-Off Duration. In the experiment, the larger $\mathrm{Ag}$ particles were filtered by using the centrifugal machine. In addition, multiple groups of onoff duration control experiments were analyzed. Besides the equal proportion comparison, the on-duration and offduration experiments were also conducted and the groups of 


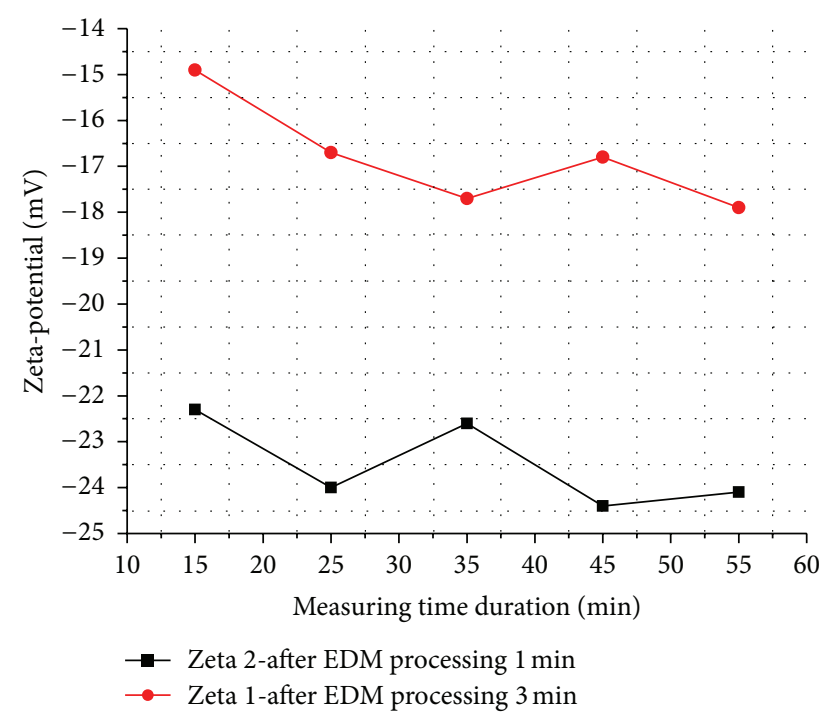

FIGURE 8: Zeta-potential analysis of silver nanofluid.

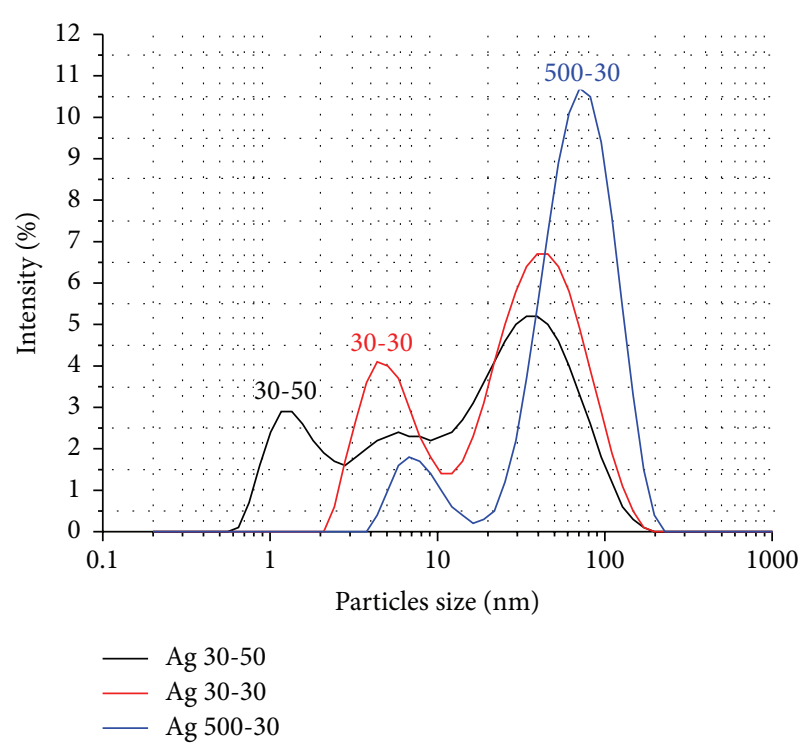

FIgURE 9: Particle size analysis of different on-off duration.

30-50, 30-30, and 500-30 were taken out for comparison. The analysis results showed that the particles of on-off duration of 500-30 were significantly larger in distribution and the most of particles were sized around $100 \mathrm{~nm}$, as shown in Figure 9; under the parameter setting of 30:50, the particle size distribution was relatively smaller, and the particle size may even be smaller than $1 \mathrm{~nm}$. Most of the particles suspending in the fluid were smaller in size, and a large amount of small particles extruded each other, resulting in the extrusion on the double electrode layer. Therefore, the Zeta-potential values were around $-35 \mathrm{mV}$ as shown in Figure 10, which was smaller than that of the larger particles sized $500: 30$. However, the value of the equal proportion control group sized 30:30 was between the two values.

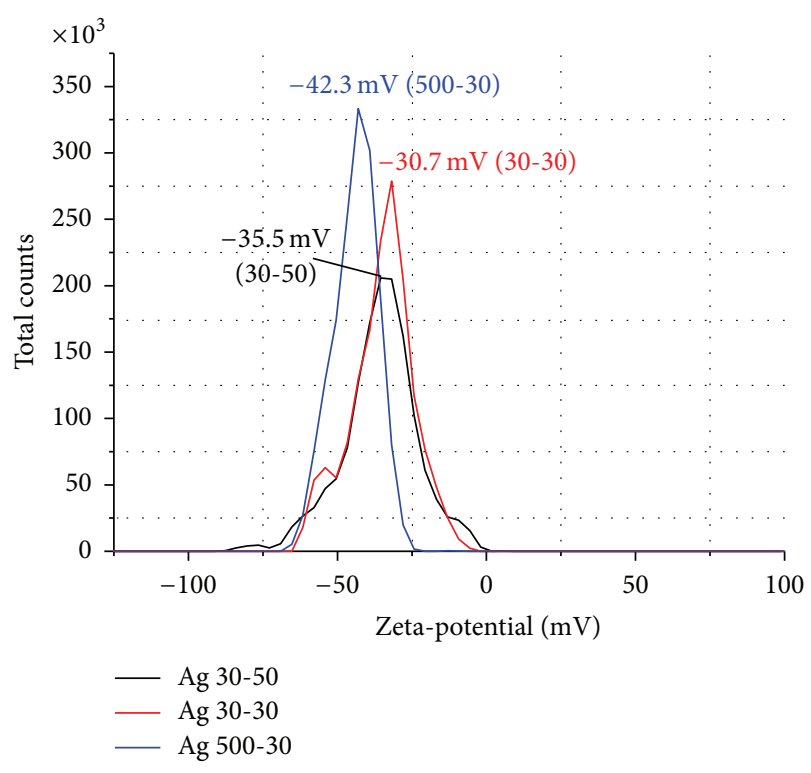

FIGURE 10: Zeta-potential analysis of different on-off duration.

\section{Conclusions}

This research focused on the preparation of silver nanofluid by the EDM system and the relationship between its process parameters and product characteristics. Based on the experimental results, the conclusions are summarized in the following points.

(1) Quantifying the relationship between the process parameters and the material removal rate (MRR) of silver electrode and the ion output rate (IOR) in the fluid and identifying the most effective process parameter condition, where on-off pulse discharge duration was around 30-30 $\mu \mathrm{s}$.

(2) For the analysis on stability of silver nanofluid, the variations of absorption spectrum, particle size, and Zeta-potential with time were observed, respectively, by UV-Vis spectroscopy, size-distribution, and Zetapotential analyzer. The experiment indicated that the silver nanofluid under EDM process showed particle and ion saturation. When the process duration was too long, stable silver nanofluid could not be obtained.

(3) The relationship of process parameters and suspensibility of silver nanofluid acquired in this study can serve as reference for future preparation of other silver nanofluids via EDM process.

(4) After the filtering of the centrifugal machine, the Ag fluid of equal proportion parameter setting of particles sized 30-30 showed better material removal rate and the same UV absorption peak value at $400 \mathrm{~nm}$. The range of the particle size distribution was about $1 \sim 200 \mathrm{~nm}$ and the concentration of the Ag fluid decreased with rising proportion. When increasing the on-duration setting, the size of the particles was 
relatively larger and the UV absorption peak value had the "Red shift." With increasing on-duration setting, the concentration of Ag fluid decreased. The deionization of the $\mathrm{Ag}$ ions enhanced under the application of the electric field. The insulation of the pure water decreased to result in a higher discharging success rate of the electrode. The concentration of discharge led to coarser and larger particles.

\section{References}

[1] N. Parkansky, O. Goldstein, B. Alterkop et al., "Features of micro and nano-particles produced by pulsed arc submerged in ethanol," Powder Technology, vol. 161, no. 3, pp. 215-219, 2006.

[2] C. H. Lo, T. T. Tsung, and H. M. Lin, "Preparation of silver nanofluid by the submerged arc nanoparticle synthesis system (SANSS)," Journal of Alloys and Compounds, vol. 434-435, pp. 659-662, 2007.

[3] K. H. Tseng, C. Y. Liao, J. C. Huang, D. C. Tien, and T. T. Tsung, "Characterization of gold nanoparticles in organic or inorganic medium (ethanol/water) fabricated by spark discharge method," Materials Letters, vol. 62, no. 19, pp. 3341-3344, 2008.

[4] Y. Y. Tsai, J. S. Su, and C. Y. Su, "A novel method to produce carbon nanotubes using EDM process," International Journal of Machine Tools and Manufacture, vol. 48, no. 15, pp. 1653-1657, 2008.

[5] N. Ichinose, Y. Ozaki, and S. Kashu, Superfine Particle Technology, 1992.

[6] D. C. Tien, K. H. Tseng, C. Y. Liao, J. C. Huang, and T. T. Tsung, "Discovery of ionic silver in silver nanoparticle suspension fabricated by arc discharge method," Journal of Alloys and Compounds, vol. 463, no. 1-2, pp. 408-411, 2008.

[7] K. H. Tseng and C. Y. Liao, "Production of silver ions from colloidal silver by nanoparticle iontophoresis system," Journal of Nanoscience and Nanotechnology, vol. 11, no. 3, pp. 1991-1995, 2011.

[8] D. C. Tien, K. H. Tseng, C. Y. Liao, and T. T. Tsung, "Colloidal silver fabrication using the spark discharge system and its antimicrobial effect on Staphylococcus aureus," Medical Engineering and Physics, vol. 30, no. 8, pp. 948-952, 2008.

[9] H. Kim, G. DeWitt, T. McKrell, J. Buongiorno, and L. W. Hu, "On the quenching of steel and zircaloy spheres in water-based nanofluids with alumina, silica and diamond nanoparticles," International Journal of Multiphase Flow, vol. 35, no. 5, pp. 427438, 2009.

[10] D. Zhu, X. Li, N. Wang, X. Wang, J. Gao, and H. Li, “Dispersion behavior and thermal conductivity characteristics of $\mathrm{Al}_{2} \mathrm{O}_{3}$ $\mathrm{H}_{2} \mathrm{O}$ nanofluids," Current Applied Physics, vol. 9, no. 1, pp. 131139, 2009.

[11] N. Sano, H. Wang, I. Alexandrou et al., "Properties of carbon onions produced by an arc discharge in water," Journal of Applied Physics, vol. 92, no. 5, p. 2783, 2002.

[12] H. Chang and S. C. Lin, "Fabrication method for a $\mathrm{TiO}_{2}$ nanofluid with high roundness and superior dispersion properties," Materials Transactions, vol. 48, no. 4, pp. 836-841, 2007.

[13] A. Calka, D. Wexler, B. Monaghan, A. Mosbah, and P. Balaz, "Rapid reduction of copper sulfide $\left(\mathrm{Cu}_{2} \mathrm{~S}\right)$ with elemental $\mathrm{Fe}$ and $\mathrm{Mg}$ using electrical discharge assisted mechanical milling (EDAMM)," Journal of Alloys and Compounds, vol. 486, no. 1-2, pp. 492-496, 2009.
[14] H. Chang, M. J. Kao, C. S. Jwo, C. G. Kuo, Y. H. Yeh, and W. C. Tzeng, "Preparation of $\mathrm{Co} / \mathrm{Ag}$ nanocompound fluid using ASNSS with aid of ultrasonic orthogonal vibration," Journal of Alloys and Compounds, vol. 504, no. 1, pp. S376-S379, 2010.

[15] W. Yu, D. M. France, J. L. Routbort, and S. U. S. Choi, "Review and comparison of nanofluid thermal conductivity and heat transfer enhancements," Heat Transfer Engineering, vol. 29, no. 5, pp. 432-460, 2008.

[16] K. H. Tsenga, C. Y. Liao, and D. C. Tien, "Silver carbonate and stability in colloidal silver: a by-product of the electric spark discharge method," Journal of Alloys and Compounds, vol. 493, pp. 438-440, 2010.

[17] K. Heiskanen, Particle Classification, Chapman and Hall, 1993.

[18] I. D. Morrison and S. Ross, Colloidal Dispersions Suspensions, Emulsions, and Foams, Wiley-Interscience, 2002.

[19] J. Jiang, G. Oberdörster, and P. Biswas, "Characterization of size, surface charge, and agglomeration state of nanoparticle dispersions for toxicological studies," Journal of Nanoparticle Research, vol. 11, pp. 77-89, 2009.

[20] T. Cosgrove, Colloid Science: Principles, Methods and Applications, Blackwell, 2005.

[21] T. Pradeep, NANO: The Essentials, McGraw-Hill Education, 2007.

[22] S. Jailani, G. V. Franks, and T. W. Healy, " $\zeta$ Potential of nanoparticle suspensions: effect of electrolyte concentration, particle size, and volume fraction," Journal of the American Ceramic Society, vol. 91, no. 4, pp. 1141-1147, 2008.

[23] Y. Liang and J. Binner, "Effect of triblock copolymer non-ionic surfactants on the rheology of $3 \mathrm{~mol} \%$ yttria stabilised zirconia nanosuspensions," Ceramics International, vol. 34, no. 2, pp. 293-297, 2008.

[24] F. Caruso, Colloids and Colloid Assemblies, Wiley-VCH, 2004.

[25] D. Kong, H. Yang, Y. Yang, S. Wei, J. Wang, and B. Cheng, "Dispersion behavior and stabilization mechanism of alumina powders in silica sol," Materials Letters, vol. 58, no. 27-28, pp. 3503-3508, 2004.

[26] J. J. McDermott and D. Velegol, "Simple fabrication of metallic colloidal doublets having electrical connectivity," Langmuir, vol. 24, no. 8, pp. 4335-4339, 2008.

[27] T. Tsuji, D. H. Thang, Y. Okazaki, M. Nakanishi, Y. Tsuboi, and M. Tsuji, "Preparation of silver nanoparticles by laser ablation in polyvinylpyrrolidone solutions," Applied Surface Science, vol. 254, no. 16, pp. 5224-5230, 2008.

[28] M. F. Meléndrez, G. Cárdenas, J. Díaz, C. Cruzat, and J. Arbiol, "Synthesis and aggregation study of tin nanoparticles and colloids obtained by chemical liquid deposition," Colloid and Polymer Science, vol. 287, pp. 13-22, 2009.

[29] S. H. Yeo, W. Kurnia, and P. C. Tan, "Critical assessment and numerical comparison of electro-thermal models in EDM," Journal of Materials Processing Technology, vol. 203, no. 1-3, pp. 241-251, 2008.

[30] K. Salonitis, A. Stournaras, P. Stavropoulos, and G. Chryssolouris, "Thermal modeling of the material removal rate and surface roughness for die-sinking EDM," International Journal of Advanced Manufacturing Technology, vol. 40, no. 3-4, pp. 316323, 2009.

[31] S. Son, H. Lim, A. S. Kumar, and M. Rahman, "Influences of pulsed power condition on the machining properties in micro EDM," Journal of Materials Processing Technology, vol. 190, no. 1-3, pp. 73-76, 2007. 

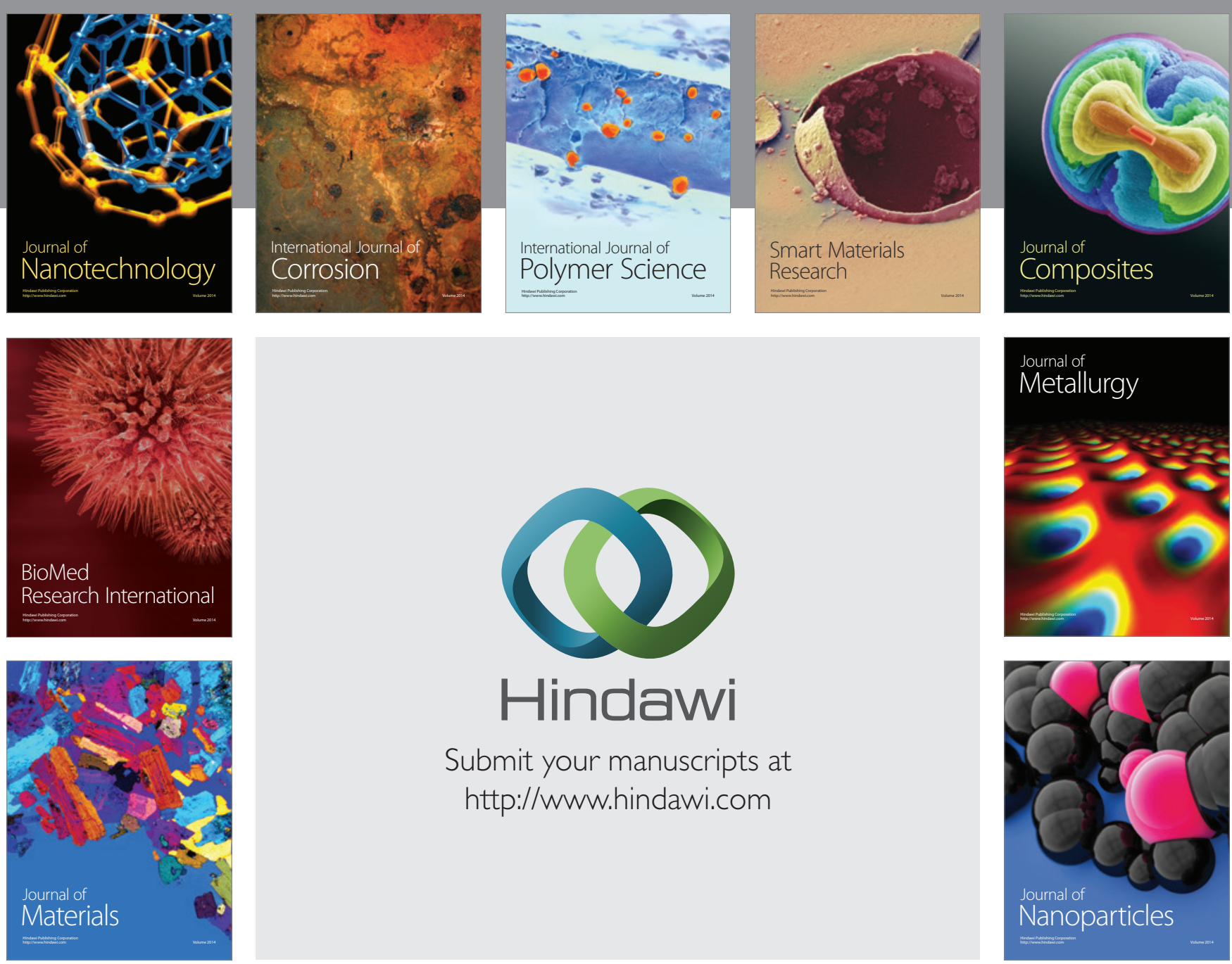

Submit your manuscripts at http://www.hindawi.com
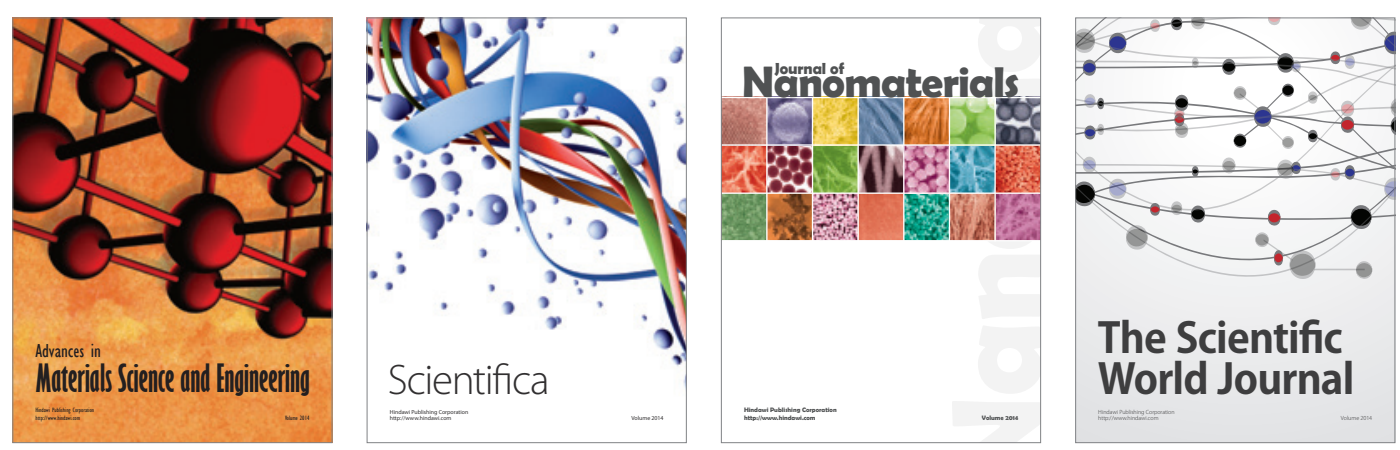

\section{The Scientific World Journal}
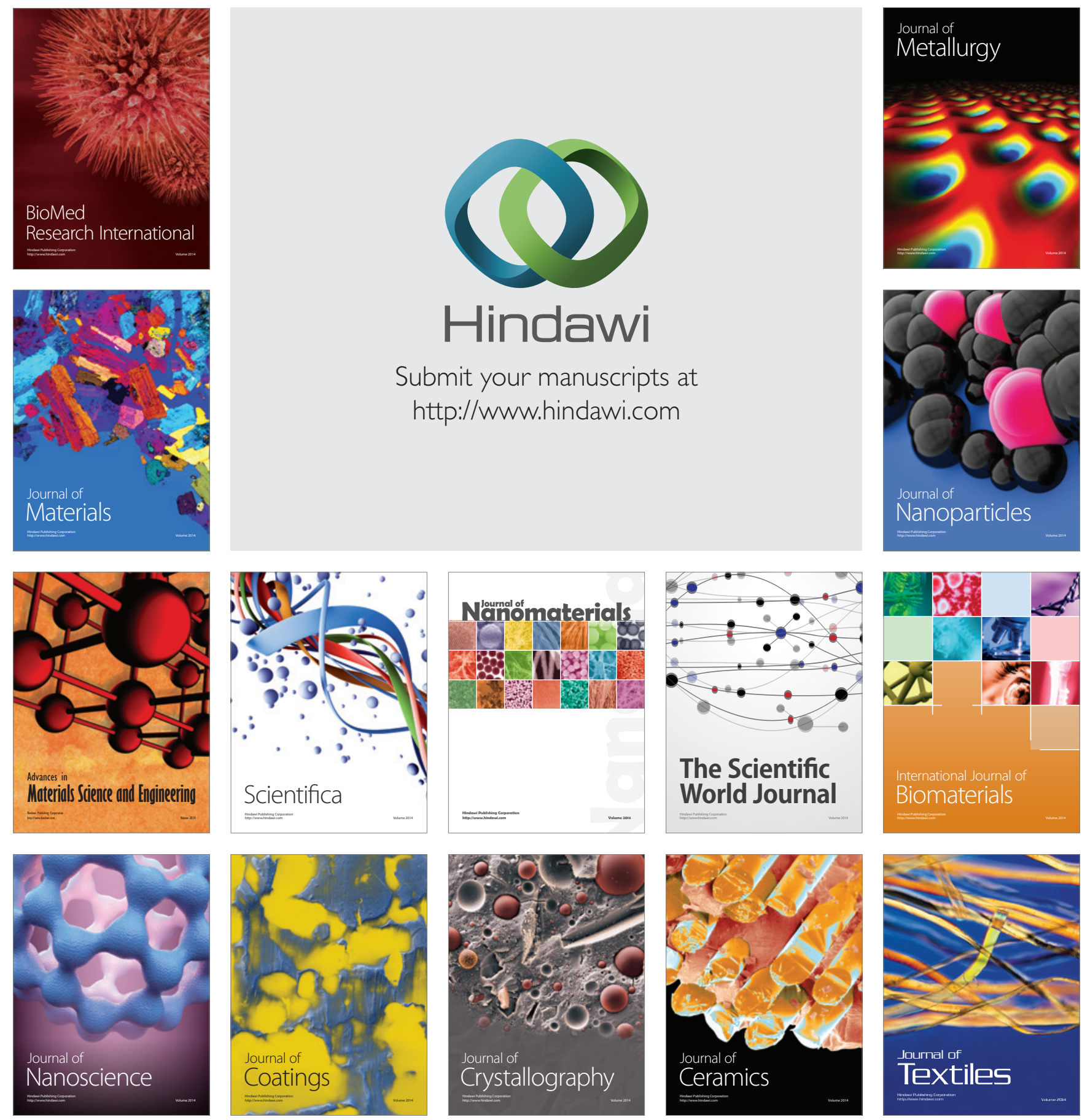